\title{
Racs based Weight Optimization and Layered Clustering-based ECOC
}

\author{
Deepak Rajak \\ School of Information \\ Technology, \\ UTD, RGPV, Bhopal, M.P., \\ India.
}

\author{
Roopam Gupta \\ School of Information \\ Technology, \\ UIT, RGPV, Bhopal, M.P., \\ India.
}

\author{
Sanjeev Sharma \\ School of Information \\ Technology \\ UTD, RGPV, Bhopal, M.P., \\ India.
}

\begin{abstract}
Error correcting output code (ECOC) is a general framework of solving a multiclass classification problem via a binary class classifier ensemble. In this paper, a new enhanced heuristic coding method, based on ECOC (RACS-ECOC) is proposed. It reiterates the following three steps until the training risk converges. The first step employs the layered clustering-based approach [1]. The approach can construct multiple different strong binary class classifiers on a given binary-class problem, so that the heuristic training process will not be stopped by some difficult binary-class problems. The second measure is the weight optimization technique [2]. It ensures the non-increasing of the heuristic training process whenever a new classier added to the ECOC ensemble. [3], here a survey and analysis of various techniques in classification and how the ECOC technique performs best among existing techniques.
\end{abstract}

In propose work instead of weighted optimization technique we would further like to work on recursive ant optimization scheme for classification

\section{Keywords}

Classifier ensemble, error correcting output codes, multiple classier systems, multiclass classification problem.

\section{INTRODUCTION}

In Machine learning Support vector machines (SVMs),neural networks, genetic algorithm etc have been used to perform the classification task but in recent time over the last decade, classier ensembles (i.e. Multiple classifier systems), such as bagging, boosting, and their mutations, have been shown to be effective approaches for solving, learning problems like classification, regression, etc. For such projects, the success of

the classifier ensembles relies strongly on a good choice of the base learners and a strong diversity among the base learners. One of the well-known classifier ensembles for solving multiclass problems is the error correcting output code (ECOC) [3]. ECOC decomposes a. multiclass problem to a serial binary-class problems.

Each binary-class problem is figured out by some binary-class classifier, such as Ada Boost and support vector machine. Given a $P$ class problem with a set of labeled samples \{( $\rho i$, Yi) $\} \mathrm{n} \mathrm{i}=1$ where $\rho \mathrm{i}$ is a dimensional sample, and $\mathrm{Yi} \in$ $\{1,2, \ldots, \mathrm{P}\}$ is the label of $\rho \mathrm{i}$, the ECO Ctriesto use Q binaryclass classifiers to address this problem. There lation between the divisions and the classifier can be extracted by a code matrix $\mathrm{M} \in\{-1,0,1\} \mathrm{P} \times \mathrm{Q}$. ECOC consists of two phases coding and decoding. In this instalment we explore the actual coding process,

ECOC tries to find a code matrix $\mathrm{M}$ for the classifier training, where the $\mathrm{p}$-th row of $\mathrm{M}$ expresses the code word of the $\mathrm{p}$-th class, denoted as cp, and the q-th column expresses the q-th classifiers, denoted as hq. If the entry $m p, q=0$, it means that hq does not take the data of the pth class into classifier training [5]. In the decoding process, conducting a test sample $\rho$ into $\mathrm{h} 1, \ldots$, HQ successively can get a test code word of $\rho$, denoted as $\mathrm{x}=[\mathrm{x} 1, \ldots, \mathrm{xQ}] \mathrm{T}$.

Given a decoding strategy $f(x, c p)$, the prediction of $\rho$ can be formulated as a minimization problem mincp $\in \mathrm{Mf}(\mathrm{x}, \mathrm{cp})$, where $M=\{c p\} Q p=1$ is the code word set. For the coding stage, there are generally two research directions for the code word design. The first direction is the problem-independent coding, design, such as the well-known one-versus-all (1vsALL) and one-versus-one (1vs1 The second direction is the problem-dependent coding, design, which looks more promising and has drawn much attention.

\section{LITERATURE REVIEW}

In this paper Xiao-Lei Zhang[1], propose an CPA based WOLC-ECOC technique, which starts with conventional ecoc and iterates it uses two steps in first step it generate strong multiple binary classifiers for most confusing binary class problem and in second step it add s new classifier by an optimized weight decoding algorithm in this way it converges some of the drawback of other techniques and perform best among these technique

In this paper A. Rahman and B. Verma [2] propose a technique of generating cluster of ensemble classifiers. That model generates a set of different level of clustering for data sets at different layers by mean of different irregular initialization of clustering parameters and trains data sets over base classifiers at different clustering levels.

It is easy to classify binary-class data but to organize multiclass data; much technique fails to generate suitable classifiers for multiclass data. For this purpose Dietterich and Bakiri propose [3] an ECOC framework technique to classify multiclass data properly. it has two parts one is encoding and another one is decoding, in encoding stage it generates different classifiers for each class based on different binary problems and in decoding stage makes decision for classification for given data based on different output.

In [4], Pujol et al. proposed a problem-dependent coding method called discriminative ECOC (DECOC). It embeds a binary decision tree to the coding; design, where each node of the tree is a powerful bipartisan of a meticulous problem. Nevertheless, the decision tree has an intrinsic defect that if a test figure is predicted wrongly by a father node, it will have no fortune to be rectified by the minor node.

To overcome this drawback, in [5], Pujol et al. Further proposed the ECOC optimizing node embedding (ECOC- 
ONE) algorithm. It begins with an initial ECOC classifier ensemble and iteratively adds the binary-class classifier that discriminates the most confusing pair of the strata to the ECOC ensemble until the desired performance is achieved. This approach repairs the intrinsic fault of the decision tree and improves the operation directly by discriminating the most confusing pair. Yet, sometimes, the most confusing pair of the strata is so "stubborn" that we cannot even find a strong binary-class classifier on the yoke.

For this problem, in [8], Escalera et al. Proposed to split the most confusing pair of the classes into various subclasses, such that a difficult learning problem can be broken down to several easier sub problems. The subclass-ECOC method puts on a new picture to the ECOC study - microstructure analysis. Nevertheless, the subclass-ECOC still uses a binary decision tree to construct the subclasses. Moreover, it has to check the scale of the subclasses, which might not be an easy task. Hither comes the question, can we use the subclass technique for the most confusing pair without employing a tree structure?

\section{RELATED WORK}

\section{Optimized Weighted Decoding:}

In [4], Escalera et al. Presented that a good decoding strategy should get each category has the same decoding dynamic range and zero decoding dynamic range bias. Then, they proposed the LW decoding algorithm.

\section{Layered Clustering-Based Approach:}

The layered clustering-based (LC) approach [1] is a special classifier ensemble. It first splits the feature space into several different subspaces by clustering, where the classification problem in each subspace is further solved by a classifier. And so it doubles the above process several times. Each independent repeat is called a layer. The LC approach contains two complementary techniques. One is the clustering in each layer. It can identify overlapping patterns that are difficult to distinguish. But it does not include any mechanism to integrate the diversity.

The other one is the layered approach. It utilizes the mechanism of the bagging and boosting to achieve the diversity between any layers for the helplessness of the first property. The layered structure, as examined in [9], Williams proves the discriminability of a classifier ensemble on a binary-class problem.

\section{Layered Clustering-Based ECOC:}

As examined in the introduction section, in [8], Escalera et al. proposed the subclass technique that splits a difficult classification problem into several easier sub-problems. Each sub problem is resolved by an independent classifier. Ultimately, the difficult problem is resolved by a classifier ensemble. Nevertheless, they used a tree structure for the splitting. In parliamentary law to inherit the advantage of the subclass technique, and in the meantime, to avoid using the decision tree for the subclass splitting, we investigate the ensemble learning for the solution as follows:

The central idea of the ensemble learning is to make a strong, diversified among the base classifiers. Broadly speaking, the methods of constructing the diversity can be grouped into four types [9].

They are the methods of

1) Manipulating the training examples,

2) Manipulating the input features,
3) Manipulating the training parameters, and

4) Manipulating the output targets.

However, aside from manipulating the outputs of the binary classifiers which is the key idea of ECOC, the diversity has been seldom referred to the ECOC study yet. To our knowledge, only in [10], prior and Wind eat manipulated different parameter settings of the base classifiers (multi-layer perception).

\section{PROPOSE METHODOLOGY}

There are various technique of ECOC ensemble but WOLCECOC perform best among of these techniques, in this paper a recursive ant colony based WOLC-ECOC is proposed, which gives more accurate results then previous one.

\section{RACS Algorithm:}

It provide a scheme to handle a large dataset and process, based on a parallel computing process "divide and conquer" which is efficient while dealing with big dataset and resolving it. The algorithm is based on the fact that the efficiency of Ant Colony applications is better for problems of smaller size having less number of cities. This occurs due to the random nature of the algorithm, in which a large number of good random decisions made on weighed choices are required to come together to construct an efficient solution and as the size of the problem increases, so do the number of decisions to be made to generate a single data. The RACS algorithm partitions the set of all nodes for a problem; say S, into two disjoint sets, say $\mathrm{S} 1$ and $\mathrm{S} 2$, and then proceeds to find solutions independently for the two sub-problems now created by focusing on reducing the lengths of the segments formed by these sets in the original data packet.

Recursive ant colony Based Optimized ECOC scheme:

Recursive Ant Colony System (RACS) algorithm is proposed, to improve the performance of Ant Colony System algorithm for large scale Data access problems by partitioning the feasible data obtained and applying the algorithm recursively on smaller pockets to find good solutions for the problems by improving the exploration efficiency of the ants. And use for propose work in this paper to enhance the efficiency of WOLC-ECOC

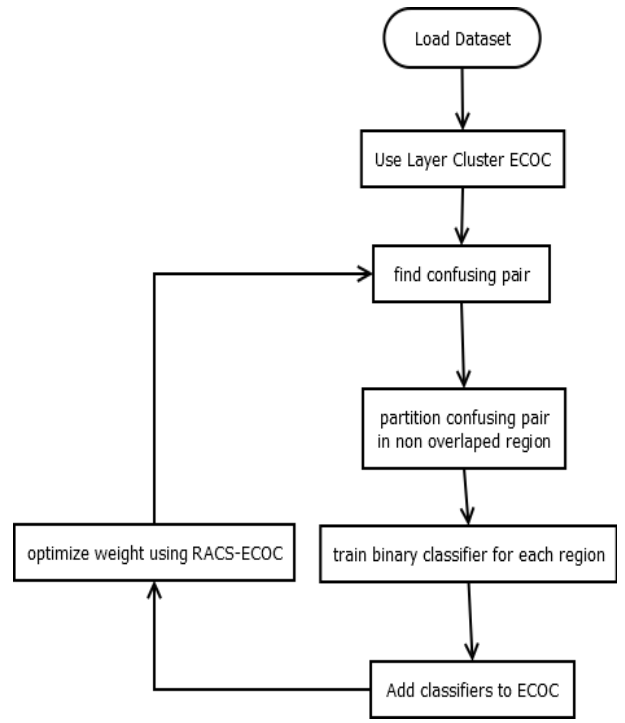

Figure1:- A Flow Diagram for proposed method 


\section{Algorithm 1 RACS-ECOC.}

Initialization: load dataset and use LC-ECOC ensemble.

1. load dataset.

2. any valid ECOC (LC-ECOC).

3. Find confusing pair of classes

4. partition confusing pair in non-overlapped regions.

5. Train a simple classier for each region.

6. Add classifier to ECOC.

7. Optimize weight using RACS system

8. repeat step 3 until risk converges.

\section{CONCLUSION AND FUTURE WORK}

In this paper, literature survey based on classification techniques is done. Various algorithms like DECOC which embeds a binary decision tree to the coding design where each node of the tree is a powerful bipartition but it has an drawback in which if any node wrongly classified than there is chance to correct it further , Subclass-ECOC it puts a new scene to the ECOC study micro structure analysis however it still uses a binary decision tree to construct the subclasses but it is difficult to control the scale of subclass with in tree, ECOC-ONE it starts with initial ECOC classifiers ensemble and iteratively adds the binary class classifier that discriminates the most confiding pair of the classes to ECOC ensemble until the desired performance reached but in this technique if there is any problem which is hard to classify it will halt the whole process, WOLC-ECOC in this technique author uses cpa based weight optimization which is time consuming and less accurate, thus here we have studied one of the recent algorithm ECOC which perform best among the various technique which were proposed so far in the field of classification, thus in future and further work we would like to perform a comparison analysis in between the existing and RAC- ECOC technique which is discussed in our paper and technique outputs should be measure and compare with the best parameters.

\section{REFERENCES}

[1] Xiao Lie Zhang "Heuristic Ternary error correcting output codes via weighted optimization and layer clustering based approaching IEEE Transactions on cybernetics, VOL. 45, NO. 2, February 2015

[2] Raman and B. Verma, "Novel layered clustering based approach for generating ensemb classifiers," IEEE Trans. Neural Netw., vol. 22, no. 5, pp. 781-792, 2011.

[3] Thomas G. Dietterich, Ghulam Bakir"Solving Multiclass Learning Problem Via Error Correcting Output Codes" Journal Of artificial Intelligence Research
2(1995) .

[4] Oriol Pujol, Petia Radovan, and Jordi Vitria, "Discriminantecoc: A heuristic method for application dependent design of error correcting output codes," IEEE Trans. Pattern Anal. Mach. Intell., vol. 28, no. 6, pp. 1007- 1012, 2006.O. Pujol, S. Escalera, and P. Radeva, "An incremental node embedding technique for error correcting output codes," Pattern Recogn., vol. 41, no. 2 pp. 713-725, 2008

[5] S. Escalera, D. M. J. Tax, O. Pujol, P. Radovan, and R P. W. Duin, "Subclass problem-dependent design for error-correcting output codes," IEEE Trans. Pattern Anal.Mach.Intell.,vol.30,no.6,pp.1041-1054,2008.

[6] X. L. Zhang, J. Wu, Z. P. Chen, and P. Lv, "Optimized weighted decoding for errorc correcting output codes," in Proc.Int.Conf.Acoustic, Speech,SignalProcess.,2012, pp. 2101-2104

[7] T. G. Dietterich and G. Bakiri, "Solving multiclass learning problems via error-correcting output codes," J. Artif. Intell. Res., vol. 2, pp. 263-286, 1995.

[8] S. Escalera, O. Poole, and P. Radeva, "On the decoding process in ternary error-correcting output codes," IEEE Trans. Pattern Anal. Mach. Intell., vol. 32, no. 1, pp. $120-134,2010$

[9] E.L.Allwein, R.E.Schapire, andY.Singer, "Reducing multiclass to binary: A unifying approach for margin classifiers," J. Mach. Learn. Res., vol. 1, pp. 113-141, 2001.

[10] T. G. Dietterich, "Ensemble methods in machine learning," in Proc. Multiple Classifier Syst., 2000, pp. 115 .

[11] Prior and T. Windeatt, "Over-fitting in ensembles of neural network classifiers within ecoc frameworks," in Proc. Multiple Classifier Syst., 2005, pp. 834-844.

[12] Nites Saharawi and Harshit Sharma "A Recursive Ant Colony for tsp" 2011 international conference on Advancements in Information Technology with workshop of ICBMG 2011.

[13] Mukesh Mann, Om Prakash SangWan"Generating and Prioriting optimal path using Ant Colony Optimization"Computational Ecology and Software, 2015.

[14] Vapnik, V.: Statistical Learning Theory. John Wiley and Sons, New York (1998)

[15] Haykin, S.: Neural Networks - A Comprehensive Foundation. Prentice Hall (1999) 